\title{
ÉVOLUTION À LONG TERME DU PEUPLEMENT PISCICOLE DU BASSIN DE LA SEINE.
}

\author{
J. BELLIARD, P. BOËT, J. ALLARDI \\ CEMAGREF, Division Qualité des Eaux, \\ 14 avenue de Saint-Mandé, 75012 Paris, France.
}

\section{RÉSUMÉ}

Des données anciennes issues de la littérature sont utilisées pour apprécier les conséquences des activités humaines sur l'évolution de la structure des communautés piscicoles du bassin de la Seine.

\section{LONG-TERM EVOLUTION OF THE FISH COMMUNITY OF THE SEINE RIVER.}

\begin{abstract}
After a brief description of the fish fauna of the Seine River basin, we attempt to measure the consequences of human activities since the beginning of the last century on the fish community structure, using historical data recorded from old literature.
\end{abstract}

\section{INTRODUCTION}

Lors des dernières glaciations, le bassin de la Seine se rattachait à un ensemble nord-ouest européen relativement isolé des autres cours d'eau centro-européens. A l'échelle biogéographique, cette situation explique le fond faunistique indigène réduit et l'importante composante en espèces amphihalines de sa faune originelle estimée entre 24 et 33 espèces (tab I; BELLIARD, 1994).

L'évolution de cette faune s'est ensuite trouvée rapidement marquée par des facteurs d'origine anthropique, qui sont progressivement devenus prépondérants en même temps qu'évoluaient les activités humaines et leurs impacts sur les cours d'eau. Aux modifications de l'environnement, survenues dès le néolithique (-5000 BP), ont succédé, depuis le Bas Moyen-âge, des modifications des cours d'eau eux-mêmes (étangs, moulins, flottage du bois, canaux de navigation, chenalisation et barrages-réservoirs...), ainsi qu'une quantité croissante de rejets polluants. A l'échelle historique, parmi les 45 espèces actuellement recensées, 7 (20 à $30 \%$ de la faune d'origine) principalement migratrices ont aujourd'hui disparu. En revanche, au moins 19 espèces résultent d'interventions humaines, d'abord d'origine européenne (pisciculture, connexions inter-bassins...), puis nordaméricaine (introductions) (fig. 1 ; tab. 1 ; BELLIARD, 1994).

Dans ce contexte fortement anthropisé, il est possible d'analyser plus en détail l'évolution structurelle du peuplement depuis le siècle dernier. Cette approche s'inspire des récents travaux menés en écologie historique sur les systèmes fluviaux européens (PETTS, 1989). Elle vise à mieux comprendre les conséquences encore méconnues des profonds bouleversements provoqués par l'intensification récente des pressions anthropiques, notamment depuis la fin du XVIIIe siècle.

\section{MATÉRIEL ET MÉTHODE}

\section{Origine et nature des données}

L'engouement pour les sciences naturelles qui a marqué le $X \mid X^{e}$ siècle s'est traduit par un grand nombre de publications destinées à dresser l'inventaire du patrimoine 
Tableau I : Liste des espèces de poissons recensées dans le bassin de la Seine ; A : espèce autochtone ; $I$ : espèce introduite ; $D$ : espèce disparue (d'après BELLIARD, 1994).

Table I : List of the fish species recorded from the Seine River basin ; A : native species ; I : introduced species ; D : extinct species (from BELLIARD, 1994).

\begin{tabular}{|c|c|c|c|}
\hline Famille & Nom commun & Statut & Code utilisé \\
\hline \multicolumn{4}{|l|}{ Acipenseridae } \\
\hline \multicolumn{4}{|l|}{ Anguillidae } \\
\hline Anguilla anguilla & Anguille & A & ANG \\
\hline \multicolumn{4}{|l|}{ Clupeidae } \\
\hline Alosa alosa & Grande alose & D & ALA \\
\hline Alosa fallax & Alose feinte & $\mathrm{D}$ & ALF \\
\hline \multicolumn{4}{|l|}{ Cyprinidae } \\
\hline Albumus albumus & Ablette & $\mathbf{A}$ & $A B L$ \\
\hline Albumoides bipunctatus & Spirlin & A & SPI \\
\hline Barbus barbus & Barbeau fluviatile & $?(\mathrm{~A})$ & $\mathrm{BAF}$ \\
\hline Abramis brama & Brème commune & A & BRB \\
\hline Blicca bjoerkna & Breme bordelière & A & BRE \\
\hline Carassius carassius & Carassin & 1 & CAS \\
\hline Carassius auratus & Carassin doré & 1 & \\
\hline Cyprinus carpio & Carpe commune & I & $\mathrm{CCO}$ \\
\hline Hypophthalmichtys molitrix & Carpe argentée & I & \\
\hline Chondrostoma nasus & Hotu & I & HOT \\
\hline Chondrostoma toxostoma & Toxostome & i & \\
\hline Gobio gobio & Goujon & A & GOU \\
\hline Leucaspius delineatus & Able de Heckel & I & \\
\hline Leuciscus cephalus & Chevaine & A & CHE \\
\hline Leuciscus leuciscus & Vandoise & A & VAN \\
\hline Leuciscus soufia & Blageon & I & \\
\hline Phoxinus phoxinus & Vairon & A & VAI \\
\hline Scardinius erythrophtalmus & Rotengle & $\mathbf{A}$ & ROT \\
\hline Rutilus rutilus & Gardon & A & GAR \\
\hline Rhodeus amarus & Bouvière & $A$ & BOU \\
\hline Tinca tinca & Tanche & A & TAN \\
\hline Pseudorasbora parva & Pseudorasbora & 1 & \\
\hline \multicolumn{4}{|l|}{ Cobitjdae } \\
\hline Cobitis taenia & Loche de rivière & A & LOR \\
\hline Nemacheilus barbatulus & Loche franche & A & LOF \\
\hline \multicolumn{4}{|l|}{ Silurid ae } \\
\hline Silurus glanis & Silure glane & I & \\
\hline \multicolumn{4}{|l|}{ Ictaluridae } \\
\hline Ictalurus melas & Poisson-chat & I & $\mathbf{P C H}$ \\
\hline \multicolumn{4}{|l|}{ Salmonidae } \\
\hline Salmo trutta trutta & Truite de mer & D & \\
\hline Salmo trutta fario & Truite fario & A & TRF \\
\hline Salmo salar & Saumon atlantique & D & SAT \\
\hline Oncorhynchus mykiis & Truite arc-en-ciel & $\mathbf{I}$ & TAC \\
\hline Salvelinus fontinalis & Saumon de fontaine & I & \\
\hline Thymallus thymallus & Ombre commun & I & OBR \\
\hline \multicolumn{4}{|l|}{ Osmeridae } \\
\hline Osmerus eperlanus & Eperlan & D & EPE \\
\hline \multicolumn{4}{|l|}{ Esocidae } \\
\hline Esox lucius & Brochet & A & BRO \\
\hline \multicolumn{4}{|l|}{ Umbridae } \\
\hline Umbra pygmaea & Umbre pygmée & I & \\
\hline \multicolumn{4}{|l|}{ Gadidae } \\
\hline Lota lota & Lote de rivière & A & LOT \\
\hline \multicolumn{4}{|l|}{ Gasterosteidae } \\
\hline Gasterosteus aculeatus & Epinoche & A & EPI \\
\hline Pungitius pungitius & Epinochette & $\mathrm{A}$ & EPT \\
\hline Cottidse & & & \\
\hline Cottus gobio & Chabot & A & CHA \\
\hline Percidae & & & \\
\hline Perca fluviarilis & Perche & $\mathbf{A}$ & PER \\
\hline Stizostedion lucioperca & Sandre & $\mathrm{I}$ & SAN \\
\hline Gymnocephalus cemua & Grémille & I & GRE \\
\hline Centrarchidae & & & \\
\hline Lepomis gibbosus & Perche soleil & I & PES \\
\hline Micropterus salmoides & Black-bass à gde b. & I & BBG \\
\hline Pleuronectidge & & & \\
\hline $\begin{array}{l}\text { Platichthys flesus } \\
\text { Petromyzonidoe }\end{array}$ & Flet & A & FLE \\
\hline Petromyzon marinus & Lamproie marine & D & LPM \\
\hline Lamperra fluviatilis & Lamproie de rivière & A & LPR \\
\hline Lampetra planeri & Lamproie de Planer & A & LPP \\
\hline
\end{tabular}




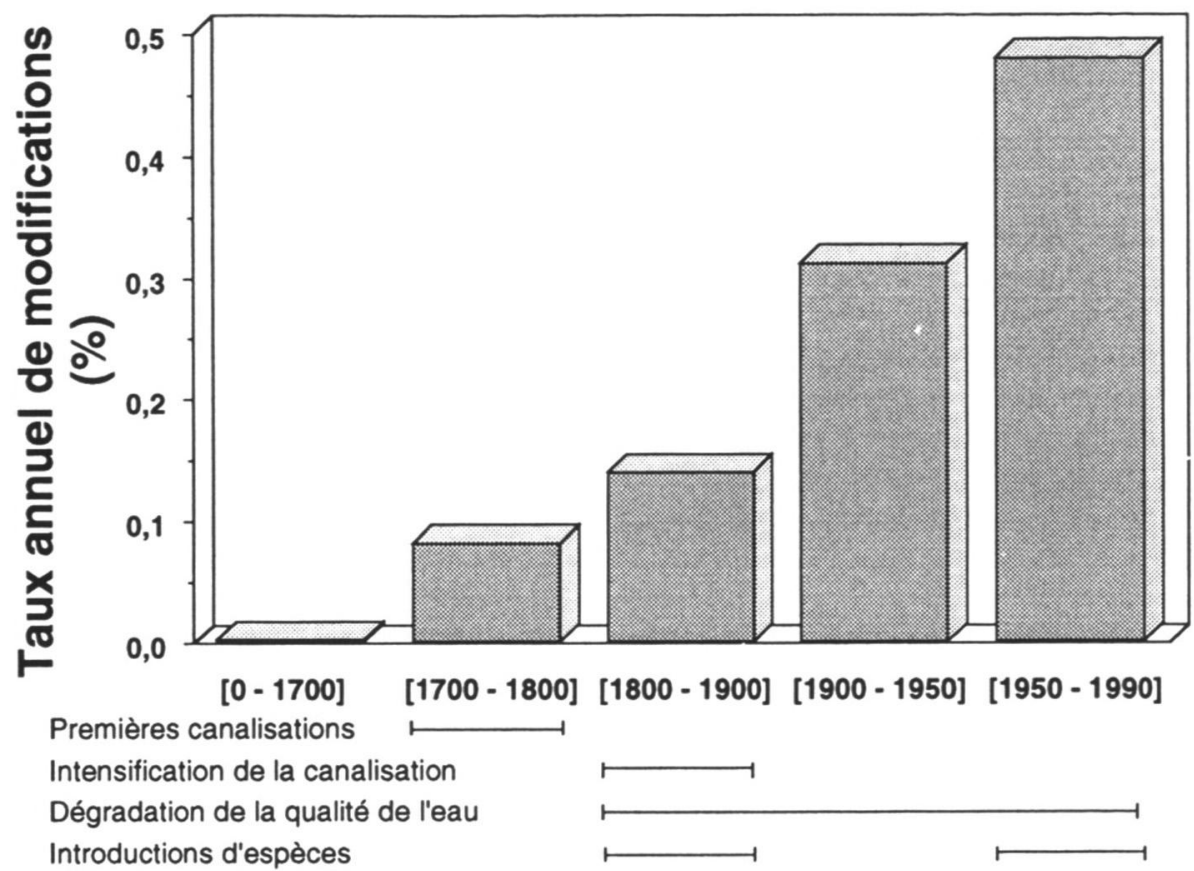

Figure 1 : Taux annuel de modifications [(sp disparues + sp introduites / sp présentes) * tps-1] de la faune piscicole de la Seine à différentes périodes historiques (d'après BELLIARD, 1994).

Figure 1 : Annual rate of modifications [(extinct $\mathbf{s p}+$ introduced $\mathrm{sp} /$ present $\mathrm{sp})$ * tps-1] of the fish fauna from the Seine River basin during different historical periods (from BELLIARD, 1994).

floristique et faunistique, à l'échelle d'un département ou d'une région. De tels inventaires concernant la faune piscicole du bassin de la Seine ont paru suffisamment fiables pour être exploités dans une perspective historique (DE SINETY, 1854 ; GADEAU DE KERVILLE, 1885, 1897 ; MOREAU, 1897, 1898 ; POPELIN, 1952 ; RAY, 1843, 1851 ; ROY, 1952).

Les informations fournies par ces travaux concernent plusieurs années et sont fondées essentiellement sur des observations de terrain ou des enquêtes auprès de pêcheurs. Elles indiquent parfois l'abondance des espèces sur des secteurs de cours d'eau assez précisément délimités.

La situation actuelle dans ces mêmes secteurs a été dressée à partir des résultats de pêches scientifiques à l'électricité (BELLIARD, 1994).

\section{Méthodologie}

Les dates et les secteurs de pêche suffisamment proches ont été regroupés, permettant ainsi de considérer plusieurs grands ensembles (tab. II). Des transformations préalables ont également été effectuées pour homogénéiser les données :

\section{- Abondance des espèces}

Le plus souvent, les données anciennes fournissent des rangs d'abondance pour chaque espèce (rare, assez rare, assez commune, commune, très commune) qui ont été respectivement notés de 1 à 5 . Parfois, l'abondance étant plus précisément caractérisée, des demi-points ont été utilisés. 

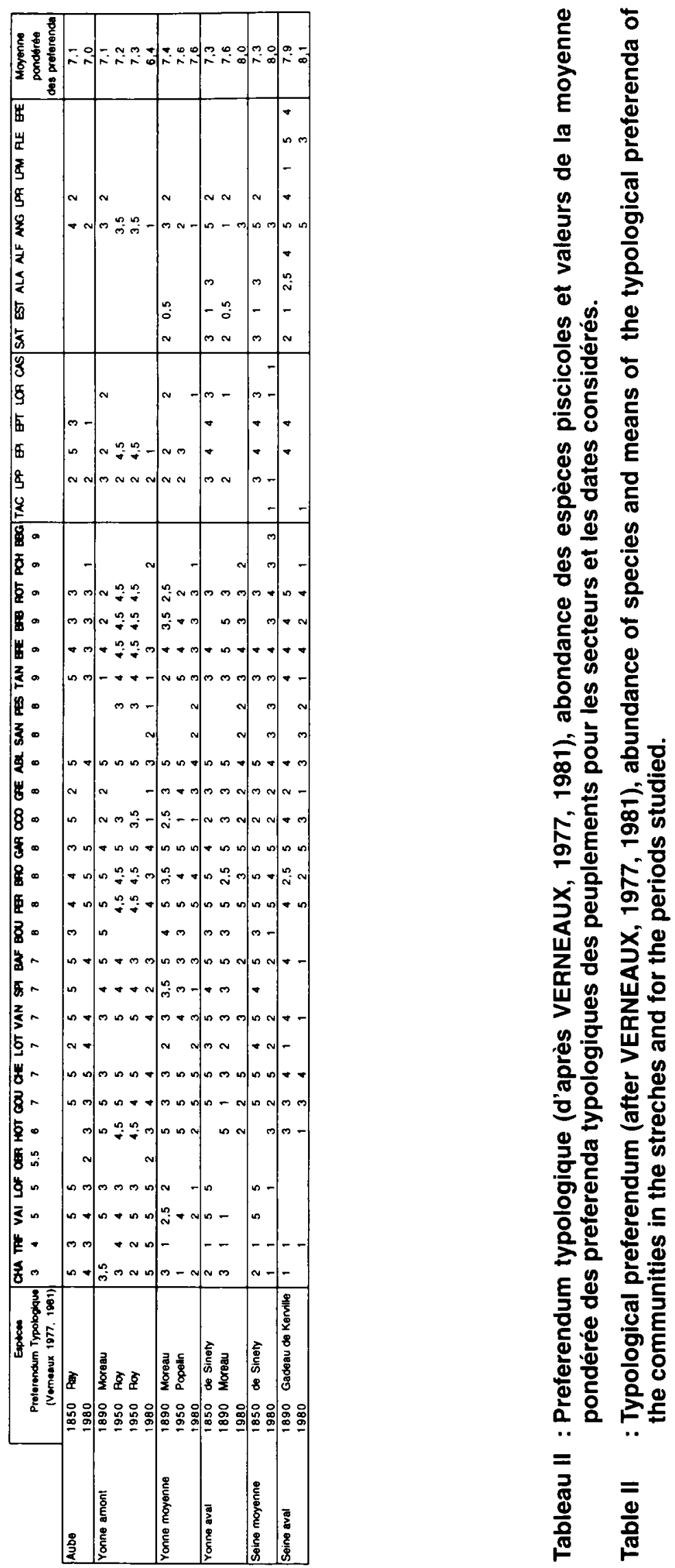
De même, pour un secteur donné, les résultats récents de pêche électrique de chaque espèce ont été transformés en note d'abondance variant de 1 à 5 . A l'espèce la plus abondante, ayant la plus grande fréquence d'occurrence et l'effectif le plus important, a été attribuée la note 5 . Inversement, une espèce rencontrée en un seul exemplaire a été affectée de la note 1. Les autres espèces ont ensuite été distribuées dans les différentes classes d'abondance en fonction de leur occurrence et de leur effectif. Bien qu'en partie subjective, cette méthode paraît cependant la plus proche possible de celle employée par les auteurs anciens.

\section{- Estimation de l'évolution globale du peuplement}

Etroitement dépendantes du gradient amont-aval des conditions mésologiques, les communautés piscicoles se caractérisent principalement par les associations d'espèces qui les composent et donc par leur position dans le gradient longitudinal (THIENEMANN, 1925 ; HUET, 1949 ; VERNEAUX, 1973, 1977, 1981).

A partir de l'étude des communautés d'insectes et de poissons du bassin du Doubs complétée plus tard par des données d'autres cours d'eau européens, VERNEAUX (1973, 1977) a identifié dix niveaux typologiques (de B0 à B9) qui se succèdent de l'amont vers l'aval d'un gradient fluvial théorique et sont caractérisés chacun par des associations d'espèces ou biocénotypes. A l'intérieur de cette structure biotypologique, cet auteur a défini le preferendum de chaque espèce piscicole (VERNEAUX, 1977, 1981).

Nous avons tenté de caractériser les peuplements étudiés sur la Seine par la moyenne des préférenda typologiques des espèces présentes pondérée par leurs abondances respectives:

$$
\sum \mathrm{l}_{\mathrm{i}} \mathrm{A}_{\mathrm{i}} / \sum \mathrm{A}_{\mathrm{i}}
$$

où, $l_{i}$ est égal au preferendum typologique de l'espèce $\mathrm{i}$ (variant théoriquement de 0 à 9 ), et $A_{i}$ son niveau d'abondance, défini comme précédemment.

Cette moyenne fournit en effet une indication concernant la position du peuplement dans le contexte amont-aval, sa valeur étant d'autant plus élevée que la proportion des espèces limnophiles est forte. Ses variations dans le temps traduisent ainsi l'évolution des peuplements pour les différents secteurs.

II convient de souligner qu'aucune espèce de poissons n'est représentée dans les trois premiers niveaux typologiques (B0 à $\mathrm{B} 2$ ), les poissons se répartissant entre les niveaux B3 et B9. Dans le cas présent le preferendum de la plupart des espèces étudiées est supérieur à 7 . Les variations de la moyenne pondérée des preferenda des peuplements sont par conséquent limitées.

Pour chaque série de données, la valeur moyenne des preferenda a été calculée sans prendre en compte les espèces rares $\left(A_{i}=1\right)$. Cette élimination des espèces rares semble en effet préférable à cause du caractère éminemment aléatoire de leur capture et parce qu'elles s'avèrent le plus souvent issues de milieux annexes (petits ruisseaux afférents, étangs...).

D'autres espèces ont également été écartées à cause de l'absence de renseignements concernant leur preferendum typologique. Elles constituent essentiellement trois groupes (tab. II) :

- les espèces migratrices ;

- la truite arc-en-ciel, la lamproie de Planer, la loche de rivière et le carassin, généralement marginaux au sein des communautés piscicoles de la Seine;

- l'épinoche et l'épinochette, dont le preferendum le long de l'axe fluvial est peu significatif.

\section{RÉSULTATS}

Les communautés piscicoles de 6 grands secteurs de cours d'eau peuvent être suivies durant des périodes s'étendant jusqu'à un siècle et demi environ, permettant ainsi d'apprécier l'évolution globale du peuplement ichtyologique du bassin de la Seine (tab. II ; fig. 2). 


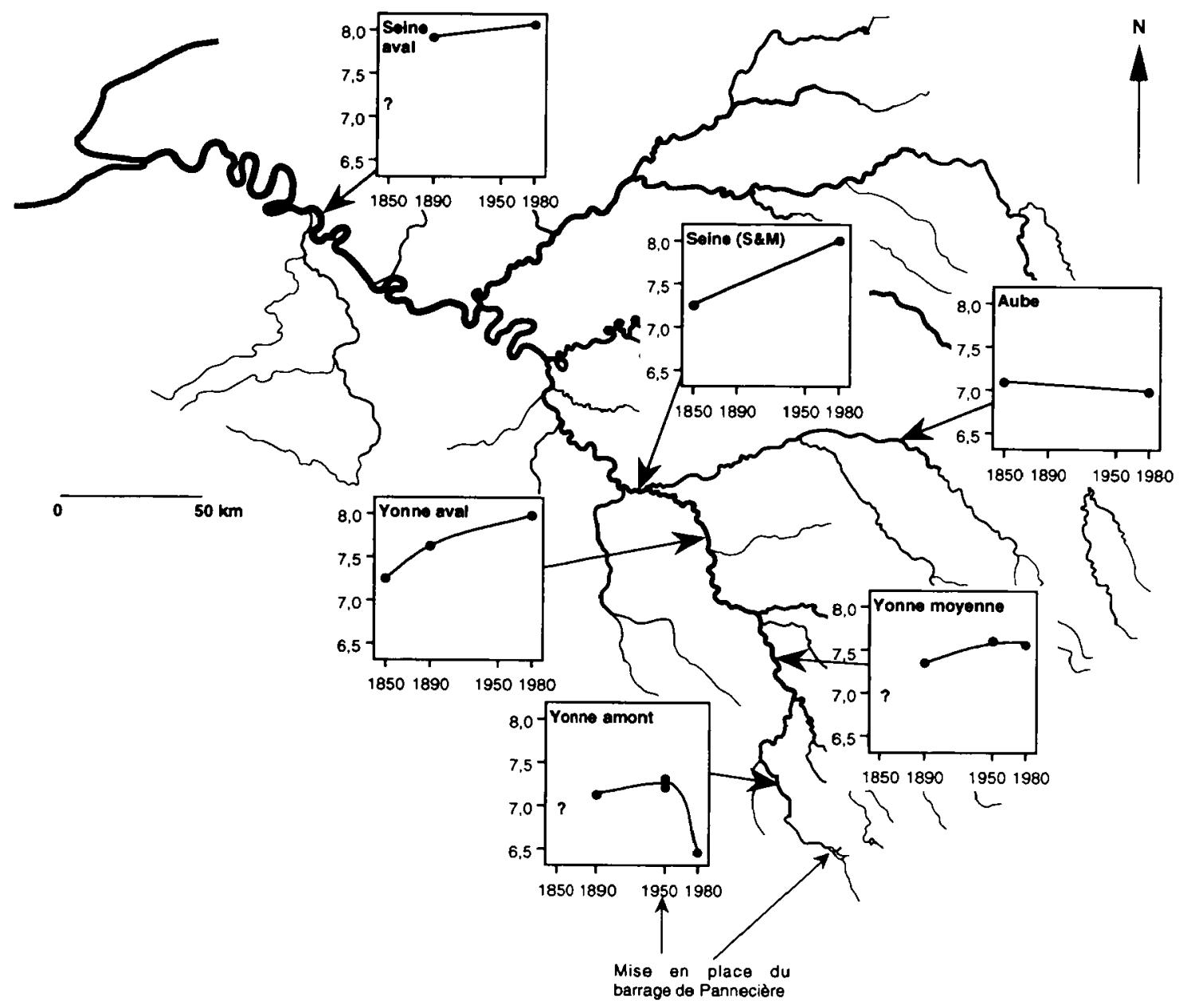

Figure 2 : Evolution historique de la structure des peuplements piscicoles de quelques secteurs du bassin de la Seine (cf. explications dans le texte).

Figure 2 : Historical changes in the structure of the fish communities in some stretches of the Seine River basin (see text for explanations).

Pour une même date donnée, la valeur de la moyenne pondérée des preferenda typologiques des peuplements augmente progressivement de l'amont vers l'aval, traduisant la prépondérance d'espèces de plus en plus limnophiles.

Globalement, depuis les dates les plus anciennes jusqu'à nos jours, une augmentation de la moyenne pondérée des preferenda typologiques est constatée sur l'ensemble du réseau. Cependant, différents schémas d'évolution se distinguent selon les secteurs de cours d'eau:

- Sur l'Aube, les valeurs observées entre 1850 et 1980 peuvent sans doute être assimilées à une situation stable.

- Sur les secteurs compris entre l'Yonne moyenne et la Seine aval, une augmentation globale est notée. Les plus importantes amplitudes sont observées sur l'Yonne aval et la Seine, secteurs pour lesquels un plus long suivi est disponible. Sur l'Yonne aval, les résultats suggèrent des changements de la structure du peuplement piscicole plus importants entre 1850 et 1890 qu'entre 1890 et 1980 . Durant cette dernière période, des changements de moindre ampleur sont constatés sur la Seine aval et l'Yonne moyenne. Sur l'Yonne moyenne, il semble même y avoir stabilisation de la situation à partir de 1950. 
- Enfin, sur l'Yonne amont, une évolution originale apparaît. Après une nette augmentation de la moyenne des preferenda entre 1890 et 1950, comparable à celle observée sur l'Yonne moyenne, suit une baisse importante qui traduit une réduction de la portion limnophile du peuplement au profit de la composante rhéophile.

\section{DISCUSSION ET CONCLUSION}

Au regard des nombreuses imprécisions qui sous-tendent la méthode, il convient bien sûr de considérer avec prudence ces différents résultats. Néanmoins, cet exercice permet de considérer le peuplement piscicole du bassin de la Seine à travers un ensemble de modifications anthropiques des écosystèmes (différents types et niveaux d'aménagements ; dégradation de la qualité des eaux) s'exprimant tant dans l'espace que dans le temps.

A elles seules, les transformations d'habitats survenues aux différentes étapes de la chenalisation du réseau fluvial peuvent expliquer une large part des changements observés sur les peuplements.

Dans le cas des secteurs de cours d'eau étudiés, la situation prévalant vers 1850 correspondait sans doute à celle de rivières encore préservées, la canalisation du fleuve et de ses affluents n'ayant en effet débuté que vers 1830. Auparavant, les rivières étaient libres ou simplement équipées de petits barrages au fil de l'eau munis de pertuis. Ensuite, la Seine a été transformée en une succession de biefs, séparés par des barrages à écluses. Vers 1850 cependant, tant la Seine moyenne que I'Yonne aval ou a fortiori l'Aube étaient encore incomplètement canalisées. En 1890, en revanche, la canalisation de toutes les parties navigables était déjà effective ; elle s'est poursuivie jusqu'à nos jours par recoupement de méandres, isolement de bras secondaires et allongement des biefs, compensé par un approfondissement du chenal. Enfin, 1950 marque l'intensification des travaux entrepris pour la régularisation du régime hydrologique du fleuve.

Ces bouleversements des écosystèmes fluviaux ont entraîné la disparition de toutes les espèces migratrices, excepté l'anguille. En outre, plusieurs espèces strictement dulcicoles ont subi des régressions plus ou moins marquées sur l'ensemble du bassin (respectivement bouvière, spirlin et loche de rivière, ainsi que loche franche, chabot, vairon, vandoise et barbeau) (tab. II).

Parmi les secteurs étudiés, celui de l'Aube, qui se remarque par la stabilité de la moyenne des preferenda typologiques des espèces pour 1850 et 1980 , a été le moins modifié (la situation de 1980 étant antérieure à la mise en service du barrage-réservoir sur son cours). Ceci tend à montrer la grande importance des aménagements physiques du fleuve sur l'évolution de sa faune piscicole. Mais cela ne signifie pas pour autant que le peuplement de ce secteur de l'Aube n'a pas évolué, certaines espèces sensibles ayant effectivement régressé.

L'évolution constatée sur la plupart des autres secteurs, caractérisée par une augmentation de la moyenne des preferenda des espèces, s'explique essentiellement par la canalisation du chenal principal qui a conduit à une réduction drastique des habitats lotiques. Au-delà, la dégradation de la qualité de l'eau, à laquelle les espèces à tendance rhéophile sont plus sensibles, a contribué à amplifier ce schéma d'évolution.

Un tout autre type de phénomène semble impliqué dans le cas de l'Yonne amont qui se singularise par une forte chute de la moyenne entre 1950 et aujourd'hui. Certes, il est possible que la description de la situation actuelle sur-représente les secteurs les plus amont, par rapport aux données anciennes. Mais il reste néanmoins certain qu'une espèce comme la truite, qui n'est pas citée par MOREAU dans le secteur de Clamecy, est maintenant bien représentée. De même, des espèces telles le rotengle ou la brème bordelière, considérées comme communes par ROY, sont aujourd'hui beaucoup plus rares.

En fait, ce changement de structure du peuplement piscicole entre 1950 et 1980 peut s'expliquer par la construction du barrage de Pannecière (1949). Publiée par ROY en 1952, la description du peuplement de l'Yonne amont s'appuie en effet sur une série d'observations antérieures, l'existence du barrage n'étant d'ailleurs pas signalée par 
l'auteur. Depuis sa mise en service, ses eaux de restitution, issues des couches profondes, ont entraîné un refroidissement artificiel durable du cours d'eau en aval de l'ouvrage. Or, si la pente est souvent considérée comme un facteur important de la structuration des peuplements piscicoles, il est bien connu que la température de l'eau reste le paramètre déterminant de la distribution à grande échelle des espèces (VERNEAUX, 1973). Un abaissement de la température est ainsi susceptible d'engendrer des changements profonds de la communauté piscicole. A l'échelle du cours d'eau, comparé au schéma théorique de HUET (1949), un décalage vers l'aval des zones piscicoles de rivières de la craie, aux eaux fraîches, est rapporté par ARRIGNON (1972). En aval de barrages, plusieurs exemples de l'influence du refroidissement des eaux de restitution entraînant une dérive des peuplements vers des composantes davantage salmonicoles ont déjà été signalés (HUET et al., 1969 ; DÉCAMPS et al., 1979 ; CEMAGREF, 1982). A l'évidence, les changements intervenus dans le peuplement piscicole de l'Yonne amont peuvent être attribués au même type de processus.

Ces résultats illustrent donc l'intérêt que présentent les données anciennes dans l'étude des peuplements piscicoles. Plus généralement, ils montrent la valeur des peuplements de poissons comme indicateur de l'évolution des systèmes fluviaux, permettant ainsi de mieux comprendre les conséquences des perturbations engendrées par les activités humaines sur leur fonctionnement. Dans le cas présent, leur réponse à long terme s'avère différente selon la nature des aménagements. Les poissons qui sont parmi les organismes aquatiques ayant été le plus précisément observés au cours des siècles passés, peuvent donc constituer de précieux éléments de référence susceptibles d'aider aux orientations en matière d'aménagement ou de réhabilitation des milieux aquatiques.

\section{REMERCIEMENTS}

Ce travail a été réalisé dans le cadre du programme CNRS PIREN-Seine intitulé «Modélisation du fonctionnement des écosytèmes fluviaux anthropisés».

\section{BIBLIOGRAPHIE}

ARRIGNON J., 1972. Zonation piscicole de quelques cours d'eau normands (France). Ver. Internat. Verein. Limnol., 18, 1135-1146.

BELLIARD J., 1994. Le peuplement ichtyologique du bassin de la Seine : rôle et signification des échelles temporelles et spatiales. Thèse Doct. Paris VI, $197 \mathrm{p}$.

CEMAGREF, 1982. Etude hydrobiologique de la Dordogne. Rapport CEMAGREF Bordeaux, Division Qualité des Eaux-Min. Environnement, Dir. Protection de la Nature, Serv. de la Pêche, Etude N³, 259 p.

DE SINETY, 1854. Notes pour servir à la Faune du département de Seine-et-Marne ou liste méthodique des animaux vivants à l'état sauvage qui se rencontrent, soit constamment, soit périodiquement ou accidentellement, dans ce département. Extrait de la Revue et Magasin de Zoologie, 2, 92-100.

DESCAMPS H., CAPBLANQ J., CASANOVA H., TOURENQ J.N., 1979. Hydrobiology of some regulated rivers in the southwest of France. In Ward J.V. and Stanford J.A., The ecology of regulated streams, 273-288, Plenum Press, New York.

GADEAU DE KERVILLE H., 1885. Aperçu de la faune actuelle de la Seine et de son embouchure (depuis Rouen jusqu'au Havre). In LENNIER G., L'estuaire de la Seine. Mémoires, notes et documents pour servir à l'étude de "l'estuaire de la Seine", 168-287, Imprimerie du Journal Le Havre n² 2, Le Havre.

GADEAU DE KERVILLE H., 1897. Faune de la Normandie. Bulletin de la Société des amis des Sciences Naturelles de Rouen, $4^{\mathrm{e}}$ Série - Trente-deuxième année (1 ${ }^{\text {er }}$ Semestre 1986), 146-656. 
HUET M., 1949. Aperçu des relations entre la pente et les populations piscicoles des eaux courantes. Scheiw. Z. Hydrol., 11 (3-4), 332-351.

HUET M., LELEK A., LIBOSVARSKY J., PENAZ M., 1969. Contribution à l'identification des zones piscicoles de quelques cours d'eau de Moravie (Tchécoslovaquie). Ver. Internat. Verein. Limnol., 17, 1103-1111.

MOREAU E., 1897. Les poissons du département de I'Yonne. Bull. Soc. Sci. Hist. Nat. Yonne, 51 (part. 2), 143-227.

MOREAU E., 1898. Les poissons du département de l'Yonne. Bull. Soc. Sci. Hist. Nat. Yonne, 52 (part. 2), 3-82.

PETTS G.E., 1989. Historical analysis of fluvial hydrosystems. In PETTS G.E., MÖLLER H. and ROUX A.L., Historical change of large alluvial rivers: Western Europe, 1-18, John Wiley \& Sons, Chichester.

POPELIN R., 1952. Le peuplement des eaux de l'Yonne moyenne. Bull. Fr. Piscic., 164, 109-114.

RAY J., 1843. Catalogue de la faune de l'Aube. Troyes, 123-137 p.

RAY J., 1851. Rapport sur la pêche fluviale dans le département de l'Aube. Soc. agric. Sci. Arts et Belles Lettres de l'Aube, Troyes, $38 \mathrm{p}$.

ROY R., 1952. Biogéographie des poissons dans la région de Nevers. Annales de la Station Centrale d'Hydrobiologie Appliquée, 4, 287-317.

THIENEMANN A., 1925. Die Binnengewässer Mitteleuropas. Eine limnologische Einführung. Die Binengewässer, Stuttgart, 1, 1-255.

VERNEAUX J., 1973. Cours d'eau de Franche-Comté (massif du Jura). Recherches écologiques sur le réseau hydrographique du Doubs. Essai de biotypologie. Thèse Doctorat Ann. Fac. Sci. Univ. Besançon, 84, $257 \mathrm{p}$.

VERNEAUX J., 1977. Biotypologie de l'écosystème “eau courante». Détermination approchée de l'appartenance typologique d'un peuplement ichtyologique. $C$. $R$. Acad. Sci. Paris, 284, sér. D, 675-678.

VERNEAUX J., 1981. Les poissons et la qualité des cours d'eau. Ann. Sci. Univ. FrancheComté, Besançon, Biol. Anim., 4ème sér. (fasc. 2), 33-41. 